\title{
pH-dependent vs. constant release of mesalazine in the treatment of ulcerative colitis: Do drug delivery concepts determine therapeutic efficacy? (Review)
}

\author{
HELMUT DEISSLER $^{1}$, HEINRICH KRAMMER ${ }^{2}$ and ANTON GILLESSEN ${ }^{3}$ \\ ${ }^{1} \mathrm{HD} / \mathrm{U}$ Scientific Consulting, D-89075 Ulm; ${ }^{2}$ Gastroenterological Practice, Center for Colon and Rectal Diseases, \\ D-68165 Mannheim; ${ }^{3}$ Department of Internal Medicine (Gastroenterology), Herz-Jesu Hospital, D-48165 Muenster, Germany
}

Received July 12, 2021; Accepted September 2, 2021

DOI: $10.3892 /$ br.2021.1472

\begin{abstract}
Inflammatory bowel diseases (IBD) have developed to become a major global health problem. Ulcerative colitis (UC) is one of two main types of IBD, and $>90 \%$ of patients suffering from mild or moderate forms of UC are treated with mesalazine, a well-tolerated and cost-effective drug. To allow oral administration, the drug has to be protected from resorption before it can reach the affected sites in the colon. The drug is therefore released from most currently used medications either constantly slow (time-dependent) or triggered by an increased $\mathrm{pH}$ during gastrointestinal transition. Both variants are widely used in clinical practice and it is surprising that they have not yet been compared directly in a large clinical study. In this overview, the evidence that may suggest preferential use of one type of mesalazine formulation over the other in general or for defined subgroups of patients is summarized and evaluated. Data from in vitro modelling of drug release and measurements of drug concentrations in colonic mucosa suggest that in many cases, constant release and $\mathrm{pH}$-dependent formulations are of similar therapeutic efficiency; however, $\mathrm{pH}$-triggered release may be superior in patients with proctitis-type UC or sites of inflammation in the proximal colon. Additionally, patients with a long gastric residence time, slow small intestinal transition, disease-related diarrhea or sensitivity to systemic adverse effects may benefit more from $\mathrm{pH}$-dependent release formulations. In general, medications based on both concepts show similar efficacies, but the $\mathrm{pH}$-dependent release formulations seem to be more robust in the treatment of a not further classified group of
\end{abstract}

Correspondence to: Professor Helmut Deissler, HD/U Scientific Consulting, Otl-Aicher-Allee 23, D-89075 Ulm, Germany

E-mail: hdulm@email.de

Abbreviations: 5-ASA, 5-aminosalicylate (mesalazine); IBD, inflammatory bowel disease(s); MMX, multi-matrix technology; UC, ulcerative colitis

Key words: 5-ASA, mesalazine, IBD, ulcerative colitis, $\mathrm{pH}$-dependent release, time-controlled release patients with UC. Future comparative clinical studies are required to clearly define the subgroups of patients that should be treated preferably with constant or $\mathrm{pH}$-dependent release formulations of mesalazine.

\section{Contents}

1. Introduction

2. Factors determining drug delivery at the colonic target site

3. In vitro models of drug release

4. Measurement of drug release in healthy individuals and patients

5. Clinical studies comparing $\mathrm{pH}$ - and time-dependent formulations

6. Conclusions

\section{Introduction}

The incidence of inflammatory bowel diseases (IBD) has increased over the past three decades worldwide with a considerable impact on health and socio-economic consequences particularly in North America and Europe. However, in view of the rapidly increasing incidence in more recently industrialized countries, e.g. in Asia, it can be considered a major global health problem (1). For mild or moderate forms of ulcerative colitis (UC), one of the two main classes of IBD, treatment with mesalazine [5-aminosalicylate (5-ASA)] is the most common first line anti-inflammatory therapy received by $>90 \%$ of patients with UC (2). This is in accordance with clinical practice guidelines published by the associations of gastroenterologists in Europe, the US and other regions of the world, with a trend to recommend treatment of even the mildest forms of colitis with 5-ASA $(3,4)$. Despite recent developments of innovative therapies with biologics and small-molecule drugs, 5-ASA maintains an outstanding role in the treatment of mild to moderate UC as it is effective, well tolerated and cost-effective $(5,6)$. Compared to alternative drugs with a similar capacity to induce remission in this largest group of UC patients, such as budesonide-multi-matrix (MMX), better tolerability still favors 5-ASA (7). 
5-ASA counteracts inflammation of the colonic mucosa by interfering with various signaling processes, of which activation of the peroxisome proliferation-activated receptor $\gamma$ (PPAR $\gamma)$ is considered the most relevant. Subsequently, downregulation of nuclear factor- $\kappa \mathrm{B}$ by PPAR $\gamma$ results in lowered amounts of effectors like prostaglandins and leukotrienes (8-10).

Prolonged therapy with 5-ASA after having achieved remission helps to maintain a disease-free state, which is an important aspect in view of the substantially higher risk of developing colon cancer associated with sustained inflammation (11). The early recognized considerable chemopreventive effect of regularly taken 5-ASA (or prodrugs) was confirmed in several studies $(12,13)$, e.g. by Eaden et al (12) who observed an $81 \%$ reduction of risk in patients with UC.

In UC, inflammatory processes are restricted to the colon, with the rectum affected in many (proctitis) cases, in contrast to Crohn's disease which potentially affects all parts of the intestine. The beneficial effect of 5-ASA clearly depends on the amount of the drug that can be delivered to the sites of inflammation (14). High concentrations in the colon can only be achieved by oral administration when 5-ASA is protected from being resorbed in the small intestine before reaching its therapeutic target sites. This can be accomplished by syntheses of pro-drugs that pass through the small intestine and are cleaved to release mesalazine by bacterial enzymes present in the colon. Due to their inferior safety profiles, drugs of this type, e.g. sulfasalazine, only play a minor role in UC therapy at present (8). The concepts implemented in the majority of currently used medications are either constant slow (time-dependent) release through an ethyl cellulose membrane, or $\mathrm{pH}$-dependent release after dissolving of an Eudragit $\mathrm{S}(\mathrm{pH}>7)$ or Eudragit $\mathrm{L}(\mathrm{pH}>6)$ coating when threshold $\mathrm{pH}$ values are exceeded during gastrointestinal transition (5). MMX technology also results in a pH-dependent release, but in addition, the drug is embedded in a matrix consisting of lipophilic and hydrophilic components (Fig. 1) (15). Whether the resulting slower dissolution of 5-ASA into the intestinal fluid is associated with a therapeutic benefit remains to be conclusively determined. This hypothesis was tested in a clinical trial in which MMX-mesalazine was more efficient than $\mathrm{pH}$-dependent release mesalazine (16). However, the daily dose of the MMX variant (4.8 g) was substantially higher than that of the $\mathrm{pH}$-dependent form (3.6 g).

It is a widely accepted view that available formulations of 5-ASA drugs have a very similar, if not identical capacity to induce or maintain remission in patients with UC. This can be concluded from overall results of clinical trials with each of the main types of 5-ASA medications, i.e. the $\mathrm{pH}$-dependent and constant release forms $(3,4,9)$. However, there are only very limited data from direct comparisons, and general considerations and in vitro simulations of gastrointestinal transition suggest that substantial differences may exist, at least for certain subgroups of patients. This possibility, which is not necessarily in contrast to the observed similar overall effectiveness of the different formulations, is discussed in the present review, in addition to a summary of relevant investigations.

\section{Factors determining drug delivery at the colonic target site}

The release of therapeutic agents acting locally in parts of the gastrointestinal tract affected by IBD, i.e. the colon in
UC patients, primarily depends on the intestinal milieu and transition times through the segments passed. It is a plausible assumption that for $\mathrm{pH}$-triggered release formulations, the $\mathrm{pH}$ profile is most relevant, whereas efficient delivery by means of constant release medications is mainly determined by transit rates. All orally administered substances are exposed after gastric emptying to an environment of increasing $\mathrm{pH}$ from the duodenum (fasted/fed $\mathrm{pH} \sim 6.2 / 5.2$ ) via the jejunum (fasted/fed $\mathrm{pH} \sim 6.9 / 6.1$ ) to the terminal ileum with a maximum $\mathrm{pH}$ of 7.5 (17). The $\mathrm{pH}$ in the ascending and transverse colon is then slightly lower (6.3) and increases again in the descending colon. These values, confirmed in numerous studies and summarized by Abuhelwa et al (18), are means determined in groups of healthy subjects showing wide inter- and intra-individual variability. At least partly, this might be due to age-dependent adjustment of the $\mathrm{pH}$ profile in the GI tract: In the distal ileum-in which pH-dependent release from such mesalazine formulations is triggered-the measured $\mathrm{pH}$ was significantly lower in individuals $>64$ years compared with young adults (19). Inter-individual differences of a number of other factors potentially co-determining the GI transition and release of drugs may also have to be taken into consideration $(20,21)$. However, in the distal small intestines of almost all individuals, the threshold $\mathrm{pH}$ to release drugs from Eudragit-S-coated formulations ( $\mathrm{pH} 7.0)$ is exceeded. In addition, food intake only affects $\mathrm{pH}$ values in the stomach and slightly in the duodenum (18). $\mathrm{pH}$ profiles in patients with mild-to-moderate UC are very similar to that of healthy subjects, with observed deviations mostly being minor increases in the distal ileum and colon (22). Lower $\mathrm{pH}$ values than in healthy individuals were measured in the ascending colons of a small number of UC patients, independent of the stage of the disease (23), but in a previous study, only in some of the most severe cases, a dramatically reduced $\mathrm{pH}$, potentially preventing complete drug release in the colon, was measured (24). Taken together, data from $\mathrm{pH}$ measurements confirmed that $\mathrm{pH}$-triggered release to the distal ileum is a robust concept of delayed drug delivery.

Delivery to and sustained therapeutic action in the colon is also determined by the transit times of the pharmaceutical products (and released 5-ASA) through the different gastrointestinal segments, which are of relevance particularly for constant release formulations. These should be taken by the patients in a fasted state, as co-ingestion of food increases the time of gastric transition. Notably, multiple-unit dosage forms (micropellets) are retained longer in the colon than conventional tablets, suggesting their superiority when a short transition caused by a disease is observed (25).

Of particular importance in the evaluation of 5-ASA delivery to sites of inflammation in patients with UC is the passage through the small intestine when a portion of it is released and resorbed, thereby lowering colonic availability and potentially causing systemic adverse effects. Although small intestinal transition (mean $\sim 3.5 \mathrm{~h}$ ) is only weakly affected by food, measured individual values were widely distributed within a 1-6 h period (25). It is unclear if these differences (co-)determine the variable individual therapeutic responses to time-dependent release formulations. Consequences of variable small intestine transition times, at least on drug release, can be studied by means of in vitro models simulating gastrointestinal transition. 

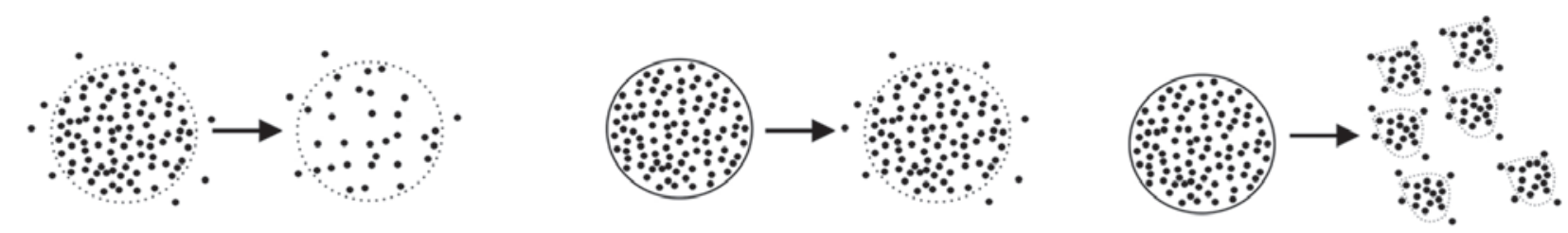

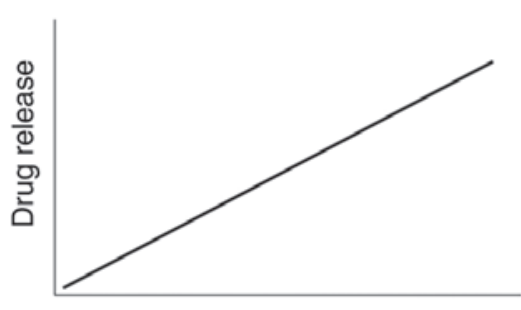

Gl transition

Time-dependent

Pentasa ${ }^{\circledR}$

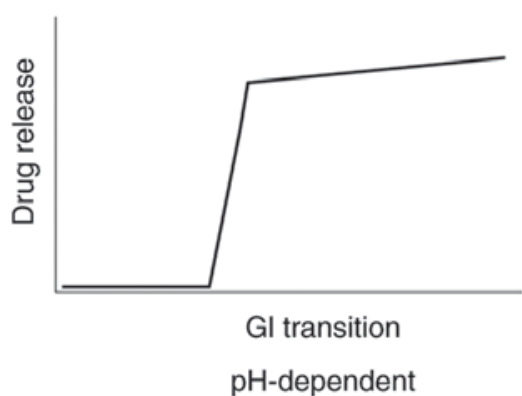

Asacol ${ }^{\circledR}$

Claversal ${ }^{\circledR}$

Salofalk ${ }^{\circledR}$

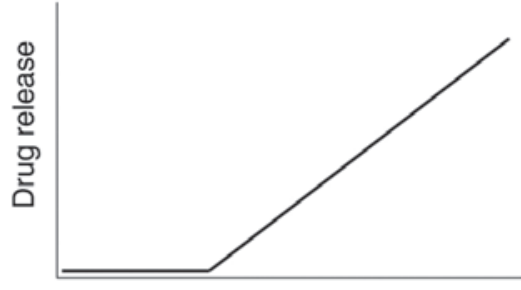

Gl transition

MMX type

Lialda ${ }^{\circledR}$

Figure 1. The common types of 5-aminosalicylate (mesalazine) formulations used to treat ulcerative colitis. Drug release from pH-dependent or MMX formulations is triggered by an increase in the luminal $\mathrm{pH}$ occurring typically in the terminal ileum. GI, gastrointestical; MMX, multi-matrix.

\section{In vitro models of drug release}

In vitro models mimic the $\mathrm{pH}$ variations during gastrointestinal passage and therefore allow studying the dissolution of solid dosage forms and associated drug release, and factors modifying these processes under defined experimental conditions (17). In an approach with a basic and a more complex buffer system Andreas et al (26) confirmed that substances typically resulting from food digestion did not alter 5-ASA release from time-dependent (Pentasa ${ }^{\circledR}$ ) or $\mathrm{pH}$-dependent $\left(\right.$ Asacol $^{\circledR}$, Salofalk $^{\circledR}$ and Claversal ${ }^{\circledR}$ ) formulations under conditions resembling those in the distal ileum. The $\mathrm{pH}$ conditions and buffers used in another investigation were too simplified to reflect gastrointestinal reality, but even this approach revealed considerable differences between several tested formulations. Of the tested constant release formulation $\left(\right.$ Pentasa $\left.^{\circledR}\right) 50 \%$ was lost in the initial phase in an acidic 'stomach-like' environment, in contrast to the $\mathrm{pH}$-triggered release variants (27). This notable effect however, was not confirmed by Karkossa and Klein (28) in their comprehensive and carefully designed in vitro comparison of different 5-ASA dosage forms. The chosen bicarbonate-based intestinal medium more accurately reflected physiological conditions, and their simulations of gastrointestinal transition were based on reliable in vivo measurements of $\mathrm{pH}$ and transit times. In addition to mean $\mathrm{pH}$ and transit time values defining an average person, individual profiles of typical and extreme subjects were used. Interesting observations made in this investigation include: i) The concept of drug release only after having passed a threshold $\mathrm{pH}$ value in the terminal ileum is realized with monolithic tablets rather than with microparticular formulations (of Salofalk ${ }^{\circledR}$ and Claversal ${ }^{\circledR}$ ), which are expected to release considerable amounts early during transition through the upper gastrointestinal tract, similar to the tested $\mathrm{pH}$-independent formulation (Pentasa ${ }^{\circledR}$ ). Avoiding loss by early resorption seems to be an advantage of the tablets, but there is a risk of insufficient delivery to sites of inflammation in the proximal colon. However, a meta-analysis of several prospective clinical trials suggested mesalazine granules to be superior to corresponding tablets in their capacity to induce remission in distal UC (29). ii) There is a clear disadvantage of constant release formulations in individuals with a long gastric residence time, resulting in extended premature release and likely loss of the active agent. iii) All tested $\mathrm{pH}$-dependent forms were almost completely discharged after 6-8 h, whereas $20-40 \%$ of the total dose still remained in the constant release formulation after the same time period of gastrointestinal transition. Of course, this portion is not available to counteract inflammation at proximal sites or in cases of accelerated transition, e.g. due to diarrhea (30). On the other hand, it is still protected from being inactivated by conversion in the colonic mucosa, and can exert its therapeutic effects in more distal regions of the colon. Comparison of simulations with several authentic gastrointestinal $\mathrm{pH}$ profiles and transition times clearly showed that for a rationale decision on the type of medication, diagnostic assessment of these parameters, e.g. by capsule endoscopy (31), and localization of the primary sites of inflammation are required. Results of in vitro investigations indicate that for UC cases with proximal sites primarily affected and fast GI transition, $\mathrm{pH}$-dependent forms may be better, in contrast to proctitis-type cases with particularly slow transition. In a clinical study however, the $\mathrm{pH}$-dependent release formulation was even more efficient in the treatment of proctitis-type UC, supporting the assumption that simulated fast transition does not reflect the clinical characteristics of a majority of patients in this group (32).

\section{Measurement of drug release in healthy individuals and patients}

Considerations based on the distinct properties of $\mathrm{pH}$-dependent and constant release formulations in their various dosage forms and of the physiological conditions they are exposed to during gastrointestinal passage, and derived in vitro models, 
are useful to predict in vivo behavior. Resulting assumptions regarding drug release in patients and associated therapeutic benefits require confirmation by results of in vivo studies. In several investigations, direct in vivo measurements of released 5-ASA and its inactive metabolite Acetyl-5-ASA (Ac-5-ASA) were performed to compare the two basic mechanisms of retarded drug release to the colon.

Determining the amount of 5-ASA in the intestinal mucosa above the threshold concentrations required to affect target molecules involved in inflammation can be regarded as a surrogate marker of therapeutic effects (33). By performing experiments with dogs, it was confirmed that sufficiently high concentrations of 5-ASA in the colonic mucosa can be achieved by oral administration of $\mathrm{pH}$-dependent release formulation Asacol ${ }^{\circledR}$, with only low amounts detected in tissue of the small intestine (34). In contrast, the time-controlled release from Pentasa ${ }^{\circledR}$ resulted in a considerable 5-ASA plasma concentration several hours after administration, confirming the advantage of galenic protection in the upper small intestine as higher plasma concentrations are associated with an increased risk of systemic side effects. Other results of these experiments are only of limited relevance, as dogs lack the ability to convert and thereby inactivate 5 -ASA by N-Acetyltransferase. D'Incà et al (35) analyzed 5-ASA concentrations in the colonic mucosa from the sigmoid region of UC patients treated with different types of formulations. Significant differences between $\mathrm{pH}$ - and time-controlled release forms were observed despite uneven $(n=73$ vs. $n=11)$ distribution of patients included. Mucosal concentrations achieved with Asacol ${ }^{\circledR}$ were substantially higher, although the administered mesalazine dose was slightly lower. Additional treatment with topical 5-ASA medications resulted in an even higher mucosal concentration, supporting the concept of combination therapy of UC affecting the terminal region of the colon.

The two prototypic formulations, Asacol ${ }^{\circledR}$ and Pentasa ${ }^{\circledR}$, were also compared in a study in which mucosal concentrations of 5-ASA and Ac-5-ASA were measured in biopsies from the rectal region (36). To compensate for differences of individually administered mesalazine doses, a mucosal concentration/dose ratio was calculated. This ratio was found to be significantly higher in patients treated with the $\mathrm{pH}$-dependent release formulation only in the subgroup suffering from mild (clinical activity index $\leq 3$ ) UC. In addition, this type of medication also resulted in a higher 5-ASA/Ac-5-ASA ratio in patients suffering from mild or more severe (clinical activity index $\geq 4$ ) UC. This is of unclear clinical relevance, but may be interpreted as a ratio of freshly delivered to already inactivated drug. However, in view of the limited number of patients, further divided in subgroups, the interesting results of this pilot study will have to be confirmed in a larger cohort. It has also been speculated that Ac-5-ASA might be a useful biomarker reflecting the therapeutic effect of 5-ASA, based on measurements in biopsies form the sigmoid colon and rectum, and correlation with disease severity in another recent pilot study (37).

\section{Clinical studies comparing pH- and time-dependent formulations}

General considerations, results based on in vitro simulations of gastrointestinal passage and measurements of concentrations of the active therapeutic agent in colonic mucosa all suggest that $\mathrm{pH}$-dependent release and constant release formulations are not completely equivalent and one or the other variant might be more beneficial for certain subgroups of patients suffering from UC. This assumption is supported by reports indicating that at least a subgroup of UC patients who responds poorly to one type of formulation benefits from switching to another sort $(38,39)$. Surprisingly, prospective clinical trials to directly compare $\mathrm{pH}$-dependent and time-dependent 5-ASA releasing formulations with large cohorts of patients with UC, allowing subgroup-specific analyses with sufficient statistical power, have not been performed yet. Literature research only revealed one large retrospective analysis of UC patients treated in Japan (40) and three small-sized prospective trials, of which two were from the same group and related $(32,41)$, and one that led to dubious results, suggesting fundamental differences between patients in Australia and Europe in their responses to time-dependent and constant release formulation (42).

Ito et al (32) compared a $\mathrm{pH}$-dependent release formulation $\left(\right.$ Asacol ${ }^{\circledR}$ ) at two different daily doses as well as a pH-independent release formulation (Pentasa ${ }^{\circledR}$ ) in their abilities to induce remission, as indicated by a lowered 'UC disease activity index', in mild to moderate UC. In an overall assessment, both types of formulations did not differ when applied at similar daily doses. Interestingly, in patients with proctitis-type UC, a significant therapeutic effect was observed with low dose (2.4 g daily) Asacol ${ }^{\circledR}$, but not with Pentasa ${ }^{\circledR}$ (2.25 g), again highlighting potential differences relevant to this particular subgroup. These two therapeutic options were compared by the same group with respect to maintenance of remission in patients with quiescent UC (41). Trends in favor of $\mathrm{pH}$-dependent release were observed, but did not reach statistical significance. Such differences however, were not revealed by retrospective analyses of data collected from patients with mild to moderate UC treated in 379 medical institutions in Japan with both formulations (40).

\section{Conclusions}

There is a great inter-individual variability in gastrointestinal $\mathrm{pH}$ profiles and transit times, determining drug release from $\mathrm{pH}$-dependent and constant release formulations. Sufficiently high concentrations of the therapeutic agent are required at the also highly variable sites of inflammation in the colon. This heterogeneity of circumstances clearly suggests different efficiencies of available types of 5-ASA formulations in subgroups of patients. Comparative clinical trials are required to define these groups and confirm results of initial studies. As suggested mainly by the summarized data from in vitro modelling and measurements of drug concentrations in the colonic mucosa, such clinical trials may reveal equivalence of $\mathrm{pH}$-dependent and constant release formulations in many cases, but superiority of the $\mathrm{pH}$-dependent release granules in patients with proctitis-type UC or sites of inflammation in the proximal colon. Other subgroups of patients that should preferably be treated with $\mathrm{pH}$-dependent release formulations include those with a long gastric residence time or slow small intestinal transition, disease-related diarrhea, and individuals who are sensitive to systemic adverse effects and therefore benefit from the galenic protection in the small intestine. In general, $\mathrm{pH}$-triggered 
release appears to be the more robust concept for delivering sufficient quantities of mesalazine to the sites of inflammation in patients with UC showing highly variable individual characteristics. However, efficacies of all approaches strongly depend on patients' compliance, which is required to ensure sustained exposure to sufficiently high amounts of the therapeutic agent.

\section{Acknowledgements}

Not applicable.

\section{Funding}

Funding from the Recordati Pharma GmbH (Ulm, Germany) supported preparation of this manuscript.

\section{Availability of data and materials}

Not applicable.

\section{Authors' contributions}

HD prepared the manuscript. HK and AG made considerable edits to the manuscript. All authors were involved in establishing the overall concept and selection of most relevant publications to be discussed. All authors have read and approved the final manuscript. Data authentication is not applicable.

\section{Ethics approval and consent to participate}

Not applicable.

\section{Patient consent for publication}

Not applicable.

\section{Competing interests}

HD received a fee from Recordati Pharma GmbH (Ulm, Germany) for his evaluation of relevant studies and preparation of the manuscript. HK and AG are consultants for Recordati and Dr. Falk Pharma GmbH (Freiburg, Germany). HK is also a consultant for Ferring GmbH (Kiel, Germany).

\section{References}

1. Ng SC, Shi HY,HamidiN, UnderwoodFE, Tang W, Benchimol EI, Panaccione R, Ghosh S, Wu JC, Chan FK, et al: Worldwide incidence and prevalence of inflammatory bowel disease in the 21st century: A systematic review of population-based studies. Lancet 390: 2769-2778, 2017.

2. Fumery M, Singh S, Dulai PS, Gower-Rousseau C, PeyrinBiroulet L and Sandborn WJ: Natural history of adult ulcerative colitis in population-based cohorts: A systematic review. Clin Gastroenterol Hepatol 16: 343-356.e3, 2018.

3. Harbord M, Eliakim R, Bettenworth D, Karmiris K, Katsanos K, Kopylov U, Kucharzik T, Molnár T, Raine T, Sebastian S, et al: Third European evidence-based consensus on diagnosis and management of ulcerative colitis. Part 2: Current management. J Crohns Colitis 11: 769-784, 2017.

4. Ko CW, Singh S, Feuerstein JD, Falck-Ytter C, Falck-Ytter Y and Cross RK; American Gastroenterological Association Institute Clinical Guidelines Committee: AGA clinical practice guidelines on the management of mild-to-moderate ulcerative colitis. Gastroenterology 156: 748-764, 2019.
5. Le Berre C, Roda G, Nedeljkovic Protic M, Danese S and Peyrin-Biroulet L: Modern use of 5-aminosalicylic acid compounds for ulcerative colitis. Expert Opin Biol Ther 20: 363-378, 2020

6. Ma C, Battat R, Dulai PS, Parker CE, Sandborn WJ, Feagan BG and Jairath V: Innovations in oral therapies for inflammatory bowel disease. Drugs 79: 1321-1335, 2019.

7. Bonovas S, Nikolopoulos GK, Piovani D, González-Lorenzo M, Pantavou K, Lytras T, Peyrin-Biroulet L and Danese S: Comparative assessment of budesonide-MMX and mesalamine in active, mild-to-moderate ulcerative colitis: A systematic review and network meta-analysis. Br J Clin Pharmacol 85: 2244-2254, 2019.

8. Desreumaux P and Ghosh S: Review article: Mode of action and delivery of 5-aminosalicylic acid-new evidence. Aliment Pharmacol Ther 24 (Suppl 1): S2-S9, 2006.

9. Nakase H: Optimizing the use of current treatments and emerging therapeutic approaches to achieve therapeutic success in patients with inflammatory bowel disease. Gut Liver 14: 7-19, 2020.

10. Stolfi C, De Simone V, Pallone F and Monteleone G: Mechanisms of action of non-steroidal anti-inflammatory drugs (NSAIDs) and mesalazine in the chemoprevention of colorectal cancer. Int J Mol Sci 14: 17972-17985, 2013.

11. Eaden JA, Abrams KR and Mayberry JF: The risk of colorectal cancer in ulcerative colitis: A meta-analysis. Gut 48: 526-535, 2001.

12. Eaden J, Abrams K, Ekbom A, Jackson E and Mayberry J: Colorectal cancer prevention in ulcerative colitis: A case-control study. Aliment Pharmacol Ther 14: 145-153, 2000.

13. Zhao LN, Li JY, Yu T, Chen GC, Yuan YH and Chen QK: 5-Aminosalicylates reduce the risk of colorectal neoplasia in patients with ulcerative colitis: An updated meta-analysis. PLoS One 9: e94208, 2014.

14. van de Meeberg MM, Schultheiss JP, Oldenburg B, Fidder HH and Huitema AD: Does the 5-aminosalicylate concentration correlate with the efficacy of oral 5-aminosalicylate and predict response in patients with inflammatory bowel disease? A systematic review. Digestion 101: 245-261, 2020.

15. Nardelli S, Pisani LF, Tontini GE, Vecchi M and Pastorelli L: MMX $^{\circledR}$ technology and its applications in gastrointestinal diseases. Therap Adv Gastroenterol 10: 545-552, 2017.

16. Ogata H, Aoyama N, Mizushima S, Hagino A and Hibi T: Comparison of efficacy of multimatrix mesalazine $4.8 \mathrm{~g}$ /day once-daily with other high-dose mesalazine in active ulcerative colitis: A randomized, double-blind study. Intest Res 15: 368-379, 2017.

17. Wahlgren M, Axenstrand M, Håkansson $\AA$, Marefati A and Lomstein Pedersen B: In vitro methods to study colon release: State of the Art and an outlook on new strategies for better in-vitro biorelevant release media. Pharmaceutics 11: 95, 2019.

18. Abuhelwa AY, Foster DJR and Upton RN: A quantitative review and meta-models of the variability and factors affecting oral drug absorption-part I: Gastrointestinal pH. AAPS J 18: 1309-1321, 2016.

19. Vertzoni M, Sulaiman S, Goumas K, Kersten E, Anlahr J, Muenster U and Reppas C: Characteristics of contents of lower intestine in the 65-74 years of age range could impact the performance of safe and efficacious modified release products. J Pharm Sci 110: 251-258, 2021.

20. Vinarov Z, Abdallah M, Agundez JAG, Allegaert K, Basit AW, Braeckmans M, Ceulemans J, Corsetti M, Griffin BT, Grimm M, et al: Impact of gastrointestinal tract variability on oral drug absorption and pharmacokinetics: An UNGAP review. Eur J Pharm Sci 162: 105812, 2021.

21. Vertzoni M, Augustijns P, Grimm M, Koziolek M, Lemmens G, Parrott N, Pentafragka C, Reppas C, Rubbens J, Van Den Abeele J, et al: Impact of regional differences along the gastrointestinal tract of healthy adults on oral drug absorption: An UNGAP review. Eur J Pharm Sci 134: 153-175, 2019.

22. Nugent SG, Kumar D, Rampton DS and Evans DF: Intestinal luminal $\mathrm{pH}$ in inflammatory bowel disease: Possible determinants and implications for therapy with aminosalicylates and other drugs. Gut 48: 571-577, 2001.

23. Vertzoni M, Goumas K, Söderlind E, Abrahamsson B, Dressman JB, Poulou A and Reppas C: Characterization of the ascending colon fluids in ulcerative colitis. Pharm Res 27: 1620-1626, 2010.

24. Fallingborg J, Christensen LA, Jacobsen BA and Rasmussen SN: Very low intraluminal colonic $\mathrm{pH}$ in patients with active ulcerative colitis. Dig Dis Sci 38: 1989-1993, 1993. 
25. Abuhelwa AY, Foster DJR and Upton RN: A quantitative review and meta-models of the variability and factors affecting oral drug absorption-part II: Gastrointestinal transit time. AAPS J 18: 1322-1333, 2016

26. Andreas CJ, Chen YC, Markopoulos C, Reppas C and Dressman J: In vitro biorelevant models for evaluating modified release mesalamine products to forecast the effect of formulation and meal intake on drug release. Eur J Pharm Biopharm 97: 39-50, 2015.

27. Goyanes A, Hatton GB, Merchant HA and Basit AW Gastrointestinal release behaviour of modified-release drug products: Dynamic dissolution testing of mesalazine formulations. Int J Pharm 484: 103-108, 2015.

28. Karkossa F and Klein S: A biopredictive in vitro comparison of oral locally acting mesalazine formulations by a novel dissolution model for assessing intraluminal drug release in individual subjects. J Pharm Sci 107: 1680-1689, 2018.

29. Leifeld L, Pfützer R, Morgenstern J, Gibson PR, Marakhouski Y, Greinwald R, Mueller R and Kruis W: Mesalazine granules are superior to Eudragit-L-coated mesalazine tablets for induction of remission in distal ulcerative colitis-a pooled analysis. Aliment Pharmacol Ther 34: 1115-1122, 2011.

30. Christensen LA, Slot O, Sanchez G, Boserup J, Rasmussen SN, Bondesen S, Hansen SH and Hvidberg EF: Release of 5-aminosalicylic acid from Pentasa during normal and accelerated intestinal transit time. Br J Clin Pharmacol 23: 365-369, 1987.

31. Koziolek M, Grimm M, Becker D, Iordanov V, Zou H, Shimizu J, Wanke C, Garbacz G and Weitschies W: Investigation of $\mathrm{pH}$ and temperature profiles in the GI tract of fasted human subjects using the intellicap $\left({ }^{\circledR}\right)$ system. J Pharm Sci 104: 2855-2863, 2015

32. Ito H, Iida M, Matsumoto T, Suzuki Y, Sasaki H, Yoshida T, Takano Y and Hibi T: Direct comparison of two different mesalamine formulations for the induction of remission in patients with ulcerative colitis: A double-blind, randomized study. Inflamm Bowel Dis 16: 1567-1574, 2010.

33. Frieri G, Giacomelli R, Pimpo M, Palumbo G, Passacantando A, Pantaleoni G and Caprilli R: Mucosal 5-aminosalicylic acid concentration inversely correlates with severity of colonic inflammation in patients with ulcerative colitis. Gut 47: 410-414, 2000.

34. Hirayama M, Toda R, Ozaki T, Hasegawa J, Nakamura T, Naraki Y,HaraguchiY,Hori Y,TanakaT,TakeiM,etal:Concentration dependence of 5-aminosalicylic acid pharmacological actions in intestinal mucosa after oral administration of a $\mathrm{pH}$-dependent formulation. Mol Pharm 8: 1083-1089, 2011.

35. D'Incà R, Paccagnella M, Cardin R, Pathak S, Baldo V, Giron MC and Sturniolo GC: 5-ASA colonic mucosal concentrations resulting from different pharmaceutical formulations in ulcerative colitis. World J Gastroenterol 19: 5665-5670, 2013.
36. Yamamoto Y, Masuda S, Nakase H, Matsuura M, Maruyama S, Hisamatsu T, Suzuki Y and Matsubara K: Influence of pharmaceutical formulation on the mucosal concentration of 5-aminosalicylic acid and $\mathrm{N}$-acetylmesalamine in Japanese patients with ulcerative colitis. Biol Pharm Bull 42: 81-86, 2019.

37. Fukuda T, Naganuma M, Takabayashi K, Hagihara Y, Tanemoto S, Nomura E, Yoshimatsu Y, Sugimoto S, Nanki K, Mizuno S, et al: Mucosal concentrations of N-acetyl-5aminosalicylic acid related to endoscopic activity in ulcerative colitis patients with mesalamine. J Gastroenterol Hepatol 35: $1878-1885,2020$

38. Kawashima K, Ishihara S, Yuki T, Onishi K, Kushiyama Y, Fujishiro H, Miyaoka Y, Yuki M, Komazawa Y, Tanimura T, et al: Therapeutic efficacy of $\mathrm{pH}$-dependent release formulation of mesalazine on active ulcerative colitis resistant to time-dependent release formulation: Analysis of fecal calprotectin concentration. Biomed Res Int 2014: 342751, 2014.

39. Yasutomi E, Hiraoka S, Yamamoto S, Oka S, Hirai M, Yamasaki Y, Inokuchi T, Kinugasa H, Takahara M, Harada K, et al: Switching between three types of mesalazine formulation and sulfasalazine in patients with active ulcerative colitis who have already received high-dose treatment with these agents. J Clin Med 8: 2019, 2019.

40. Nagahori M, Kochi S, Hanai H, Yamamoto T, Nakamura S, Omuro S, Watanabe M and Hibi T; OPTIMUM Study Group: Real life results in using 5-ASA for maintaining mild to moderate UC patients in Japan, a multi-center study, OPTIMUM Study. BMC Gastroenterol 17: 47, 2017.

41. Ito H, Iida M, Matsumoto T, Suzuki Y, Aida Y, Yoshida T, Takano Y and Hibi T: Direct comparison of two different mesalamine formulations for the maintenance of remission in patients with ulcerative colitis: A double-blind, randomized study. Inflamm Bowel Dis 16: 1575-1582, 2010.

42. Gibson PR, Fixa B, Pekárková B, Bátovský M, Radford-Smith G, Tibitanzl J, Gabalec L, Florin TH and Greinwald R: Comparison of the efficacy and safety of Eudragit-L-coated mesalazine tablets with ethylcellulose-coated mesalazine tablets in patients with mild to moderately active ulcerative colitis. Aliment Pharmacol Ther 23: 1017-1026, 2006.

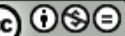

This work is licensed under a Creative Commons Attribution-NonCommercial-NoDerivatives 4.0 International (CC BY-NC-ND 4.0) License. 